\title{
Breast Reconstruction with the Free TRAM or DIEP Flap: Patient Selection, Choice of Flap, and Outcome
}

Maurice Y. Nahabedian, M.D., Bahram Momen, Ph.D., Gregory Galdino, M.D., and Paul N. Manson, M.D.

Baltimore and College Park, Md.

Recent reports of breast reconstruction with the deep inferior epigastric perforator-(DIEP) flap indicate increased fat necrosis and venous congestion as compared with the free transverse rectus abdominis muscle (TRAM) flap. Although the benefits of the DIEP flap regarding the abdominal wall are well documented, its reconstructive advantage remains uncertain. The main objective of this study was to address selection criteria for the free TRAM and DIEP flaps on the basis of patient characteristics and vascular anatomy of the flap that might minimize flap morbidity. A total of 163 free TRAM or DIEP flap breast reconstructions were performed on 135 women between 1997 and 2000. Four levels of muscle sparing related to the rectus abdominis muscle were used. The free TRAM flap was performed on 118 women, of whom 93 were unilateral and 25 were bilateral, totaling 143 flaps. The DIEP flap procedure was performed on 17 women, of whom 14 were unilateral and three were bilateral, totaling 20 flaps. Morbidities related to the 143 free TRAM flaps included return to the operating room for 11 flaps ( 7.7 percent), total necrosis in five flaps (3.5 percent), mild fat necrosis in 14 flaps (9.8 percent), mild venous congestion in two flaps (1.4 percent), and lower abdominal bulge in eight women (6.8 percent). Partial flap necrosis did not occur. Morbidities related to the 20 DIEP flaps included return to the operating room for three flaps (15 percent), total necrosis in one flap ( 5 percent), and mild fat necrosis in two flaps (10 percent). Partial flap necrosis, venous congestion, and a lower abdominal bulge were not observed. Selection of the free TRAM or DIEP flap should be made on the basis of patient weight, quantity of abdominal fat, and breast volume requirement, and on the number, caliber, and location of the perforating vessels. Occurrence of venous congestion and total flap loss in the free TRAM and DIEP flaps appears to be independent of the patient age, weight, degree of muscle sparing, and tobacco use. The occurrence of fat necrosis is related to patient weight $(p<$ 0.001 ) but not related to patient age or preservation of the rectus abdominis muscle. The ability to perform a sit-up is related to patient weight $(p<0.001)$ and patient age $(p$ $<0.001)$ but not related to preservation of the muscle or intercostal nerves. The incidence of lower abdominal bulge is reduced after DIEP flap reconstruction $(p<$ $0.001)$. The DIEP flap can be an excellent option for properly selected women. (Plast. Reconstr. Surg. 110: 466, 2002.)



Current methods of microvascular breast reconstruction using abdominal tissue include the free transverse rectus abdominis muscle (TRAM) and deep inferior epigastric perforator (DIEP) flaps. These techniques have evolved in an attempt to reduce the morbidity related to the abdominal wall and improve the aesthetics of the reconstructed breast. However, because of the complexity of these microvascular procedures, there are inherent risks that include total flap loss, partial flap loss, fat necrosis, and abdominal bulge or hernia. A review of the literature is provided in Table I. The incidence of complications after free TRAM reconstruction related to the breast ranges from 8 to 13 percent, and that related to the abdomen ranges from 1 to 82 percent. ${ }^{1-8}$ Early reports with the DIEP flap were encouraging, demonstrating an equally low flap loss rate with improved strength and contour of the abdominal wall. ${ }^{9-11}$ However, more recent reports after DIEP flap reconstruction have demonstrated an increased incidence of breastrelated morbidity that includes fat necrosis ranging from 6 to 62.5 percent and venous congestion in 4 percent. ${ }^{12-15}$ This has led many to question whether the added time and effort required in dissecting the DIEP flap is of ben-

From the Johns Hopkins Medical Institutions, Plastic and Reconstructive Surgery, and the Department of Natural Resource Sciences, University of Maryland. Received for publication June 15, 2001; revised November 20, 2001. 
TABLE I

Review of Literature

\begin{tabular}{|c|c|c|c|c|c|}
\hline Author & Year & Flap & Fat Necrosis (\%) & Venous Congestion (\%) & Bulge $(\%)$ \\
\hline \multirow[t]{2}{*}{ Blondeel et al. ${ }^{10}$} & 1997 & Free TRAM & NA & NA & $2 / 20(10)$ \\
\hline & & DIEP & NA & NA & $0 / 18(0)$ \\
\hline \multirow[t]{2}{*}{$\operatorname{Kroll}^{26}$} & 1998 & Pedicle TRAM & $18 / 67(26.9)$ & NA & NA \\
\hline & & Free TRAM & $4 / 49(8.2)$ & NA & NA \\
\hline Blondeel $^{13}$ & 1999 & DIEP & $6 / 100(6)$ & NA & $1 / 87(1.1)$ \\
\hline Hamdi et al. ${ }^{11}$ & 1999 & DIEP & $3 / 50(6 \%)$ & NA & $2 / 42(4.8)$ \\
\hline \multirow[t]{2}{*}{ Blondeel et al. ${ }^{12}$} & 2000 & Free TRAM & NA & $0 / 271$ & NA \\
\hline & & DIEP & $\mathrm{NA}$ & $5 / 240(2.1)$ & NA \\
\hline \multirow[t]{3}{*}{ Kroll $^{14}$} & 2000 & Free TRAM & $/ 279(12.9)$ & NA & NA \\
\hline & & DIEP (grou & & NA & NA \\
\hline & & DIEP (grou & & NA & NA \\
\hline
\end{tabular}

NA, not applicable.

efit when compared with the muscle-sparing free TRAM flap.

The purpose of this study is to establish selection criteria for the free TRAM and DIEP flaps on the basis of patient characteristics and the vascular anatomy of the flap and to provide an outcome analysis. Flap morbidity is analyzed on the basis of the degree of sparing of the rectus abdominis muscle, tobacco use, patient age, and patient body weight. Abdominal morbidity is analyzed on the basis of the amount of muscle used and sparing of the intercostal innervation and its association with the ability to perform a sit-up and occurrence of a lower abdominal bulge. More specifically, we intended to quantify whether venous congestion, total flap loss, fat necrosis, lower abdominal bulge, or the ability to perform a sit-up would be related to the patient's age, weight, tobacco use, nerve sparing, or the muscle-sparing method used during the operation. An algorithm for selecting the free TRAM or DIEP flap will be provided.


Anatomic Basis of the Free TRAM and DIEP Flaps

The anatomy, preoperative markings, technique of elevation, and choice of recipient vessels for the free TRAM and DIEP flaps have been previously described. ${ }^{16-19}$ The defining and differentiating features of the free TRAM and DIEP flaps include the presence or absence of rectus abdominis muscle and the number of perforating vessels perfusing the flap. The amount of rectus abdominis muscle incorporated into a free TRAM flap is variable and ranges from a short length and entire width of the muscle to a small $2 \times 2-\mathrm{cm}$ segment of muscle. The latter type of free TRAM flap is known as a muscle-sparing free TRAM flap and also incorporates a small segment of anterior rectus sheath (Fig. 1). The free TRAM flap contains a variable number of perforators (generally three to six). The DIEP flap incorporates no muscle or anterior rectus sheath, and the number of perforators included generally ranges from one to three (Fig. 2). The anatomic features of the deep inferior epigastric artery and vein and its perforators have been described be means of cadaver dissections and color-flow duplex scanning. ${ }^{20-23}$ The average mean diameter of the deep inferior

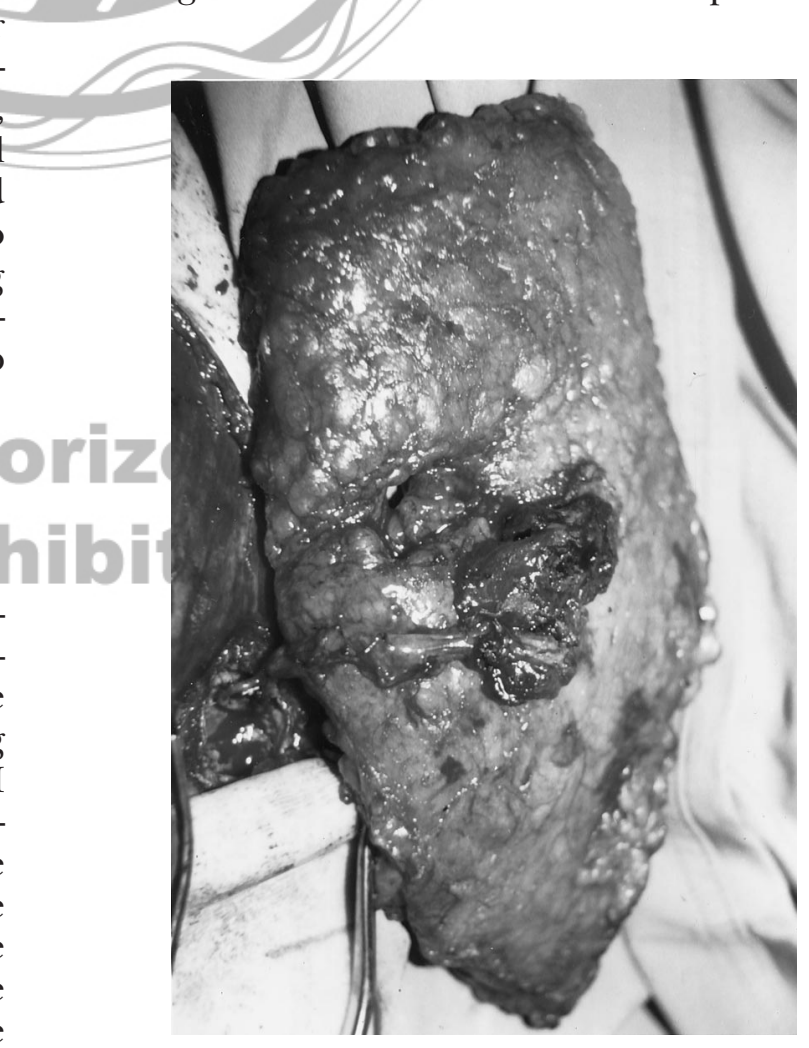

FIG. 1. Free TRAM flap with small central segment of rectus abdominis muscle (MS-2). 


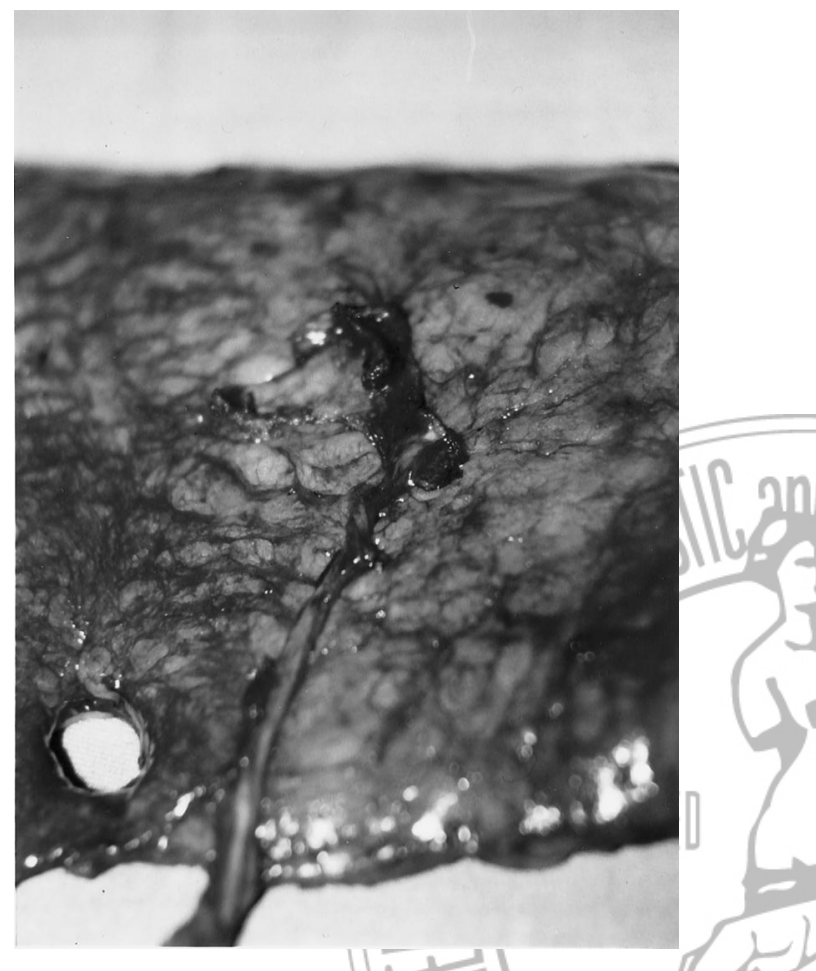

FIG. 2. DIEP flap (MS-3). Note that the rectus abdominis muscle and anterior rectus sheath are not incorporated into the flap.

epigastric artery is $3.6 \mathrm{~mm}$ (range, 2.8 to 5 $\mathrm{mm}$ ). The number of perforators with a caliber greater than $1 \mathrm{~mm}$ ranges from one to three. The majority of perforators are located within $8 \mathrm{~cm}$ of the umbilicus.

\section{Patient and Flap Selection}

The decision to use a free TRAM or DIEP flap is made on the basis of preoperative and intraoperative factors. ${ }^{24-29}$ Although a woman may be a good candidate for a DIEP flap on the basis of preoperative factors, the delivery of a DIEP flap might not be possible because of intraoperative factors. Important preoperative factors include breast size, breast volume, body habitus, quantity and quality of abdominal skin and fat, and tobacco use. Intraoperative factors include the number, caliber, and location of the perforators.

The size and volume of the natural breast are major determinants that can influence the choice of flap. Breast volume can be estimated on the basis of physical parameters or calculated on the basis of three-dimensional imaging techniques. ${ }^{29}$ On the basis of our past experience (unpublished data), women with a breast volume less than $1000 \mathrm{cc}$ are potential candidates for DIEP or free TRAM flap recon- struction, whereas women with a breast volume greater than $1000 \mathrm{cc}$ are better candidates for the free TRAM flap. This is because largevolume $(>1000 \mathrm{cc})$ breast reconstruction may be at increased risk for flap-related morbidity because of inadequate vascular perfusion. Although the DIEP flap has been demonstrated clinically to provide adequate perfusion based on one or two perforators, its benefit for largevolume reconstruction has not been established. Therefore, it may be helpful to estimate or calculate the breast volume to choose a flap that will be capable of adequate perfusion for the entire flap.

For the majority of women in this study, breast volume was estimated on the basis of the primary surgeon's personal experience. For 22 women (32 breasts), breast volumes were calculated preoperatively using the Rainbow three-dimensional digital imaging system (Genex Technologies, Inc., Kensington, Md.) to assist with the decision-making process. ${ }^{29}$ The imaging was performed during initial consultation. Postoperative yolume measurements were not obtained. Mean preoperative breast volume for the 32 breasts was $493 \mathrm{cc}$ (range, 185 to $1109 \mathrm{cc}$ ). Mean preoperative volume for the six breasts that were reconstructed using a DIEP flap was 493 cc (range, 311 to $753 \mathrm{cc}$ ) and the mean preoperative volume for the 26 breasts that were reconstructed using a free TRAM flap was $492 \mathrm{cc}$ (range, 185 to $1109 \mathrm{cc}$ ). In the remaining 113 women that included 14 DIEP flaps and 117 free TRAM flaps, breast volumes were not obtained. In these women, the decision was made on the basis of body habitus and qualitative estimation of tissue requirements.

-Assessment of the abdomen may also influence the choice of flap. In general, morbidly obese women with a large abdominal pannus are discouraged from proceeding with abdominal flap reconstruction; however, women with a mild to moderate amount of abdominal tissue are candidates for free TRAM or DIEP flap reconstruction..$^{25}$ Women with a history of tobacco use may be better candidates for free TRAM reconstruction to include more perforators and minimize the morbidity related to tobacco usage. ${ }^{24}$ Duplex Doppler ultrasound has been used preoperatively to assess the quality and quantity of perforators. ${ }^{20}$ This test is especially useful for women with a large body habitus or those having prior abdominal operations. 
Prior abdominal operations may influence the choice of free TRAM or DIEP flap reconstruction, and may preclude the use of an abdominal flap. Operations that transect the system of periumbilical perforators, such as abdominoplasty, are contraindications to performing reconstruction using an abdominal flap. However, there can be incisions on the abdomen that do not contraindicate the use of an abdominal flap. Incisions located in the lower transverse abdomen (Pfannenstiel), midline abdomen, and lateral abdomen are not contraindications to abdominal flap reconstruction provided the inferior epigastric vessels are intact. A lower midline incision (between the pubis and umbilicus) allows for use of a hemiflap only, whereas an upper midline incision (between the umbilicus and xiphoid) will allow for use of the entire flap.

Intraoperative factors that influence the choice of free TRAM or DIEP flap reconstruction include the number, caliber, and location of the perforators. This assessment is made at the level of the anterior rectus sheath after the separation of the flap from the anterior rectus sheath, leaving the major perforators intact. Reconstruction with a DIEP flap is usually possible after identification of one or two perforators with a minimum caliber of $1.5 \mathrm{~mm}$. For breast reconstruction requiring less than $750 \mathrm{~g}$ of tissue, one perforator is usually sufficient. For reconstructions requiring between 750 and $1000 \mathrm{~g}$, two perforators are generally used. Conversion to a free TRAM flap, incorporating an island of fascia, perforators, and muscle, is performed when the criteria for perforator selection are not observed.

The quantity of abdominal tissue to reconstruct a breast is determined on the basis of qualitative information. The surface dimensions of the flap are determined on the basis of the preoperative topography of the breast and the mastectomy specimen to obtain similar size and shape. The mastectomy specimen is evaluated before making the abdominal incision and an outline is delineated on the abdomen. The thickness of the mastectomy specimen sometimes exceeds that of the abdominal flap, and in these situations the lateral portion of the flap (zone III) in infolded and sutured to the chest wall after the microvascular anastomosis to increase breast projection. Flap weight or volume is not measured.

Outcome is analyzed on the basis of the morbidity related to the flap and the abdomen.
Flap morbidity includes return to the operating room within the first 48 hours, total flap necrosis, partial flap necrosis, fat necrosis, and venous congestion. Total flap loss is defined as complete necrosis of the skin and fat and is usually caused by compromised circulation at the anastomosis. Partial flap necrosis is defined as loss of a portion or segment of the cutaneous and fat components because of insufficient circulation distal to the anastomosis. Fat necrosis is defined as a hardening of a portion of the fat component with complete viability of the cutaneous component. The various degrees of fat necrosis were assessed clinically and graded as mild ( $<5$ percent), moderate ( 5 to 20 percent), or severe $(\$ 20$ percent). Imaging studies to quantify the amount of fat necrosis were not performed. Wenous congestion is defined as a circulatory imbalance in which the arterial inflow exceeds the venous outflow. Mild venous congestion is defined as sluggish venous outflow that is nonprogressive and self-limiting without requiring operative intervention and possibly requiring medicinal leech therapy. Severe venous congestion requires operative intervention.

Abdominal morbidity is assessed by means of strength and contour. Abdominal strength is measured as the ability to perform a sit-up. Quantitative analysis using isokinetic testing was not performed in this study but has been performed in other studies evaluating the free TRAM and DIEP flaps. ${ }^{1,6,9-11,13}$ Abdominal contour was assessed by physical examination and patient response. The first author (M.Y.N.) performed all examinations to minimize any variation in the examination process. The physical examination consisted of visual assessment of contour and palpation of the abdominal wall to determine whether a fascial defect or laxity was present. Quantitative analysis using magnetic resonance imaging or computed tomographic scanning was not performed.

Patient satisfaction was assessed subjectively through follow-up visits for women undergoing reconstruction with a free TRAM or DIEP flap and a questionnaire for women undergoing reconstruction with the DIEP flap. All women were asked during follow-up visits whether they could perform sit-ups, if they were pleased with the abdominal contour, or if they had any dissatisfaction. Questionnaires were distributed to all women after breast reconstruction with the DIEP flap; these questionnaires asked four questions: (1) Were you happy with your ab- 
dominal contour before surgery? (2) Are you happy with your abdominal contour after surgery? (3) Is your abdominal strength after surgery less than, equal to, or better than your abdominal strength before surgery? (4) Can you perform sit-ups? No questionnaire was distributed to the women after free TRAM reconstruction because many of the questions had been answered during follow-up visits.

\section{Study Design}

This is a retrospective review of a single surgeon's (M.Y.N.) experience between October 1997 and December 2000 following breast reconstruction with the free TRAM and DIEP flaps. A total of 143 free TRAM flaps and 20 DIEP flap breast reconstructions were performed on 135 women. Various methods of muscle sparing (MS) and nerve sparing have been used that represent an evolution in the authors' technique to minimize the morbidities related to the abdominal wall and reconstructed breast (Table II). All attempts are made to preserve the lateral intercostal innervation when the muscle-sparing techniques are used. The neural anatomy of the rectus abdominis muscle has been previously described. ${ }^{30,31}$

Demographic and clinical variables are listed in Table III. The muscle-sparing free TRAM flaps are categorized as MS-1 or MS-2. Preservation of the intercostal nerve is defined as maintaining nerve continuity to the point of entry into the lateral segment of rectus abdominis muscle. Tobacco use is defined as use of more than 10 cigarettes per day. Cessation of tobacco use for greater than 3 months placed women in a "quit" or nonsmoker status.

\section{Statistical Analysis}

The response variables consisted of venous congestion, total flap loss, fat necrosis, lower abdominal bulge, and the ability to perform a sit-up. These variables were defined as a binary (class) response taking "yes" or "no" values. The explanatory variables included two quan-

TABLE II

Classification of Muscle Sparing

\begin{tabular}{cl}
\hline Muscle-Sparing Technique & \multicolumn{1}{c}{ Definition (Rectus Abdominis) } \\
\hline MS-0 & Full width, partial length \\
MS-1 & Preservation of lateral segment \\
MS-2 & Preservation of lateral and \\
& medial segments \\
MS-3 (DIEP) & Preservation of entire muscle \\
\hline
\end{tabular}

TABLE III

Demographic and Clinical Variables Related to Free TRAM and DIEP Flap Reconstruction

\begin{tabular}{lcc}
\hline \multicolumn{1}{c}{ Variable } & Free TRAM Flap & DIEP Flap \\
\hline Women & 118 & 17 \\
Unilateral & 93 & 14 \\
Bilateral & 25 & 3 \\
Flaps & 143 & 20 \\
Mean age (yr) & 49.4 & 46.4 \\
Age range (yr) & $25-75$ & $39-64$ \\
Mean weight (lb) & 176 & 147 \\
Weight range (lb) & $121-270$ & $115-190$ \\
Tobacco use (women) & 23 & 2 \\
Immediate reconstruction (flaps) & 133 & 18 \\
Delayed reconstruction (flaps) & 10 & 2 \\
Muscle-sparing flaps & 105 & 20 \\
Nerve sparing & 88 & 20 \\
Thoracodorsal anastomosis & 138 & 9 \\
Internal mammary anastomosis & 5 & 1 \\
Single perforator flap & $\mathrm{NA}$ & 17 \\
Double perforator flap & $\mathrm{NA}$ & 3 \\
Mean follow-up (mo) & 19.5 & 8.2 \\
Follow-up range (mo) & $5-44$ & $4-15$ \\
\hline
\end{tabular}

titative or continuous variables that included the patient's age and weight and three categorical or class variables that included tobacco use, nerve sparing, and the muscle-sparing method. Tobacco use and nerve sparing were defined as a "yes" or "no" response, whereas the muscle-sparing treatment was defined as having four levels (MS-0, MS-1, MS-2, and MS-3).

The binary response variables were individually related to the explanatory variables using the GENMOD procedure of the SAS System. ${ }^{32}$ This procedure is uniquely suited for categorical data analysis in which a binary response is to be related to a combination of categorical and continuous variables. The binomial distribution and logit options of the GENMOD procedure were used. Pairwise comparisons of the four muscle-sparing methods were made when the analysis of variance resulted in an overall significant effect for the muscle-sparing factor. Pairwise comparisons were made using the "estimate" option of the GENMOD procedure.

\section{RESULTS}

An analysis of factors related to morbidity for the free TRAM and DIEP flaps is provided in Table IV. An analysis of the factors associated with the occurrence of fat necrosis is listed in Table V. A comprehensive statistical analysis is provided in Table VI. 
TABLE IV

Flap Morbidities Related to the Free TRAM and DIEP Flaps

\begin{tabular}{lcc}
\hline \multicolumn{1}{c}{ Morbidity } & Free TRAM Flap & DIEP Flap \\
\hline Return to OR (flaps) & 11 & 3 \\
Indication for return to OR & 7 & 2 \\
Venous thrombosis & 2 & 0 \\
Arterial thrombosis & 1 & 0 \\
Avulsion injury & 1 & 1 \\
Hematoma & 6 & 2 \\
Flap salvage & 5 & 1 \\
Total flap necrosis & 0 & 0 \\
Partial flap necrosis & 2 & 2 \\
Venous congestion & 14 & \\
Fat necrosis &
\end{tabular}

Flap morbidity related to the free TRAM flap included return to the operating room for 11 flaps (7.7 percent), total necrosis in five flaps (3.5 percent), mild fat necrosis in 14 flaps (9.8 percent), and mild venous congestion in two flaps (1.4 percent). Mild fat necrosis $(<5$ percent) was clinically evident in three of 38 MS-0 flaps (7.9 percent), four of 31 MS-1 flaps (12.9 percent), and seven of 74 MS-2 flaps (9.5 percent). Mild venous congestion, not requiring surgical exploration, was treated successfully with medicinal leech therapy. Other complications included lower extremity deep venous thrombosis in one woman (0.87 percent).

Abdominal morbidity was analyzed as the occurrence of an abdominal bulge or the inability to perform sit-ups (Table VII). A lower abdominal bulge occurred in eight of 118 women (6.8 percent) and included four of 25 women (16 percent) following a bilateral free TRAM flap and four of 93 women (4.3 percent) following a unilateral free TRAM flap. No woman developed an upper bulge or hernia. The abdominal bulge was repaired in all eight women. Intraoperative findings included severe attenuation of the anterior rectus sheath in six women and mild laxity of the sheath in two women. In two women in the MS-2 group, intraoperative findings also included severe atrophy and fibrosis of the remaining muscle. A nerve-sparing technique was used for one of these women and a non-nerve-sparing technique was used for the other woman. Techniques of bulge repair included sheath plication in two women and onlay Marlex mesh reinforcement in six women. There has been no bulge recurrence at a minimum 1-year follow-up. An analysis of factors related to the
TABLE V

Associated Factor Analysis of Women Developing Fat Necrosis

\begin{tabular}{cclclc}
\hline Patient & $\begin{array}{c}\text { Age } \\
(\mathrm{yr})\end{array}$ & Tobacco & $\begin{array}{c}\text { Body } \\
\text { Weight (lb) }\end{array}$ & Flap & $\begin{array}{c}\text { Muscle-Sparing } \\
\text { Technique }\end{array}$ \\
\hline 1 & 38 & Never & 185 & TRAM & MS-0 \\
2 & 53 & Never & 145 & TRAM & MS-1 \\
3 & 57 & Never & 172 & TRAM & MS-0 \\
4 & 57 & Never & 195 & TRAM & MS-1 \\
5 & 53 & Quit & 215 & TRAM & MS-1 \\
6 & 52 & Quit & 190 & TRAM & MS-2 \\
7 & 63 & Never & 180 & DIEP & MS-3 \\
8 & 48 & Never & 270 & TRAM & MS-2 \\
9 & 41 & Never & 170 & TRAM & MS-2 \\
10 & 62 & Never & 145 & TRAM & MS-1 \\
11 & 51 & Never & 162 & TRAM & MS-2 \\
12 & 51 & Never & 160 & TRAM & MS-0 \\
13 & 48 & Quit & 175 & TRAM & MS-2 \\
14 & 58 & Active & 155 & TRAM & MS-2 \\
15 & 49 & Never & 148 & DIEP & MS-3 \\
16 & 38 & Active & 245 & TRAM & MS-2 \\
\hline
\end{tabular}

occurrence of an abdominal bulge is listed in Table VIII.

Although abdominal strength following the free TRAM was not quantified, 86 percent (102 of 118) reported the ability to perform sit-ups (Table VII). This included 87 of 93 (94 percent) women following unilateral breast reconstruction and 15 of 25 (60 percent) women following bilateral breast reconstruction. Despite the ability to perform sit-ups, most women [92 of 118 (78 percent)] have reported that postoperative abdominal strength was less than preoperative abdominal strength.

\section{DIEP Flap}

Flap morbidity related to the DIEP reconstruction included return to the operating room for three flaps (15 percent), total necrosis in one flap ( 5 percent), and fat necrosis in two flaps (10 percent). Partial flap necrosis and mild venous congestion were not observed. The sole flap loss occurred as a result of inability to restore adequate flow following prolonged venous thrombosis. Other complications included lower extremity deep venous thrombosis in one woman following bilateral reconstruction (5 percent).

Abdominal morbidity related to the DIEP flap has not been observed (Table VII). No woman complained of a lower abdominal bulge or had evidence of a lower bulge or hernia on the basis of physical examination. Satisfaction with preoperative abdominal contour was expressed in two of 17 women (12 percent), whereas satisfaction with postopera- 
TABLE VI

Statistical Analysis

\begin{tabular}{lccccc}
\hline \multicolumn{1}{c}{ Response } & Weight & Age & Tobacco Use & Muscle Sparing & Nerve Sparing \\
\hline Venous congestion & 0.341 & 0.289 & 0.278 & 0.541 & NA \\
Fat necrosis & $<0.001$ & 0.189 & 0.796 & 0.254 & NA \\
Total flap loss & 0.165 & 0.306 & 0.194 & 0.766 & NA \\
Ability to perform sit-ups & $<0.001$ & $<0.001$ & 0.193 & 0.205 & 0.596 \\
Lower abdominal bulge & 0.63 & $<0.02$ & 0.36 & $<0.001$ & NA \\
\hline
\end{tabular}

NA, not applicable.

tive abdominal contour was expressed in 17 of rence of a lower abdominal bulge was signifi17 women (100 percent). Comparing postop- $n$ n cantly $/(p<0.05)$ and positively related to bierative and preoperative abdominal strength lateral reconstruction and to the muscledemonstrated less postoperative strength in one of 17 women ( 5.9 percent), equal postoperative strength in 14 of 17 women (82.3 percent), and greater postoperative strength in two of 17 women (11.8 percent). The ability to perform sit-ups from a supine position was noted preoperatively in 16 of 17 women (94.1 percent) and postoperatively in the same 16 women. The only woman notable to perform a sit-up was a 68-year-old following bilateral reconstruction.

\section{Statistical Analysis}

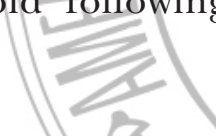

Fat necrosis was significantly and positively related to the patient's weight $(p<0.001)$; however, it does not appear to be related to age, tobacco use, or muscle sparing. Venous congestion does not appear to be related to age, weight, tobacco use, or degree of muscle sparing. Total flap loss was not related to age, weight, tobacco use, or muscle sparing. The ability to perform sit-ups was significantly but negatively related to the patient's age $(p<$ $0.001)$ and weight $(p<0.001)$; however, no significant association was detected between the ability to perform sit-ups and tobacco use, muscle sparing, or nerve sparing. The occursparing method. The DIEP flap (MS-3) decreased bulging significantly $(p<0.01)$ when compared with the MS-0 or MS-1 technique. However, there was no significant difference between MS-3 and MS-2 in terms of bulging. Pairwise comparisons of MS-0, MS-1, and MS-2 did notindicate any significant difference in terms of bulging. Lower abdominal bulge was significantly $(p<0.02)$ and positively related to age; however, it was not related to tobacco use or weight. An algorithm for selecting the free TRAM or DIEP flap is provided in Table IX.

3

Over the past decade, there has been an evolution in breast reconstruction using muscle-sparing abdominal flaps to minimize abdominal morbidity. ${ }^{1,2,7,9-11,13,18,20,28,33,34}$ This is because the muscle serves as a carrier for the inferior epigastric artery and vein and usually does not contribute to the shape or volume of the reconstructed breast. In addition, minimizing the amount of muscle removed may reduce the morbidity to the abdominal wall as it relates to strength and contour. However, when preservation of the abdominal musculature re-

\section{OTABLE VII}

Analysis of Factors Related to Abdominal Morbidity

\begin{tabular}{|c|c|c|c|c|c|c|c|c|}
\hline Type of Flap & Muscle Sparing & No. of Patients & No. of Flaps & Nerve Sparing & $\begin{array}{l}\text { No. of } \\
\text { Bulges }\end{array}$ & Bulge (\%) & No. of Sit-Ups & Sit-Ups (\%) \\
\hline \multirow[t]{3}{*}{ Unilateral free TRAM } & MS-0 & 30 & 30 & 0 & 1 & 3.3 & 26 & 87 \\
\hline & MS-1 & 23 & 23 & 20 & 1 & 4.3 & 22 & 96 \\
\hline & MS-2 & 40 & 40 & 32 & 2 & 5 & 39 & 98 \\
\hline Total & & 93 & 93 & 52 & 4 & 4.3 & 87 & 94 \\
\hline \multirow[t]{3}{*}{ Bilateral free TRAM } & MS-0 & 4 & 8 & 0 & 2 & 50 & 1 & 25 \\
\hline & MS-1 & 4 & 8 & 8 & 1 & 25 & 1 & 25 \\
\hline & MS-2 & 17 & 34 & 28 & 1 & 5.9 & 13 & 76 \\
\hline Total & & 25 & 42 & 36 & 4 & 16 & 15 & 60 \\
\hline Unilateral DIEP & MS-3 & 14 & 14 & 14 & 0 & 0 & 14 & 100 \\
\hline Bilateral DIEP & MS-3 & 3 & 6 & 6 & 0 & 0 & 2 & 67 \\
\hline Total & & 135 & 163 & 108 & 8 & 5.9 & 118 & 87 \\
\hline
\end{tabular}


TABLE VIII

Analysis of Factors Related to Lower Abdominal Bulge

\begin{tabular}{ccccc}
\hline Patient & Age $(\mathrm{yr})$ & Flap & Muscle Sparing & Nerve Sparing \\
\hline 1 & 49 & UFT & MS-0 & No \\
2 & 48 & UFT & MS-1 & No \\
3 & 62 & UFT & MS-2 & Yes \\
4 & 45 & UFT & MS-2 & Yes \\
5 & 58 & BFT & MS-0 & No \\
6 & 59 & BFT & MS-0 & No \\
7 & 54 & BFT & MS-1 & Yes \\
8 & 50 & BFT & MS-2 & Yes \\
\hline
\end{tabular}

UFT, unilateral free TRAM; BFT, bilateral free TRAM. rior epigastric vascular system that may provide the dominant flow to the abdominal skin and fat. ${ }^{12}$ Ligation of these vessels may result in a transient arterial and venous insufficiency and explain the observed venous congestion and fat necrosis. Other explanations include tobacco use, advanced patient age, obesity, inadequate number and caliber of perforators from the deep inferior epigastric system, sacrifice of dominant perforators, sparing of the rectus abdominis muscle, increased zone II requirements, and poor patient selection. . $^{1424,25,27}$

Whether to use a free TRAM or DIEP flap for breast reconstruction is decided on the basis of the physical characteristics of the patient and the anatomic characteristics of the flap. A free TRAM flap can be used for breast reconstruction of almost any volume; however, it is especially indicated when the tissue requirement exceeds $1000 \mathrm{cc}$. This is determined on the basis of the primary author's experience with pedicle and free TRAM flaps (unpublished data). The rationale justifying the use of the free TRAM flap is that a small segment of muscle and anterior rectus sheath is necessary to obtain enough perforators to sufficiently perfuse the fasciocutaneous component of the flap. The DIEP flap can also be used for breast reconstruction with large volume requirements; however, its use is dependent on the caliber, location, and number of perforators. In general, the DIEP flap is useful for breast reconstructions that do not exceed $1000 \mathrm{cc}$. Our algorithm is to use a single perforator DIEP flap when the estimated tissue requirement is less than $750 \mathrm{cc}$ and a double perforator DIEP flap when the estimated tissue requirement is between 750 and $1000 \mathrm{cc}$. The rationale justifying the use of the DIEP flap is that the perforating vessels are of sufficient caliber to adequately perfuse the fasciocutaneous component and that these perforators can be safely separated from the rectus abdominis muscle. However, the final decision to proceed with DIEP flap reconstruction should be made intraoperatively after the perforators have been visualized. When the perforators are of adequate caliber (at least $1.5 \mathrm{~mm}$ ), a DIEP flap can be performed. When no dominant perforator is located, a muscle-sparing free TRAM should be performed.

This study has demonstrated that the incidence of fat necrosis and venous congestion occurring within the free TRAM and DIEP flaps is not related to tobacco use, patient age, 
or degree of muscle sparing; however, it is related to patient weight. As patient weight increases, the volume requirements of the flap to obtain symmetry may also increase. Larger flaps can be predisposed to develop fat necrosis in light of increased vascular demand with a limited vascular supply. Although the calculation of body mass index would have been beneficial, the association between fat necrosis and body weight as it relates to flap volume requirements is still valid. The incidence of fat necrosis for the free TRAM and DIEP flaps is not significantly different in properly selected women.

Analysis of abdomen strength demonstrates that the DIEP flap does not result in an increased ability to perform sit-ups when compared with the free TRAM flap. Maximal preservation of the rectus abdominis muscle and the intercostal nerve does not appear to provide an additional advantage. Although quantitative testing of abdominal strength was not performed, the ability to perform sit-ups was demonstrated in 86 percent of women after free TRAM reconstruction and in 94 percent of women after DIEP flap reconstruction. We recognize that the ability to perform sit-ups does not accurately reflect abdominal strength because of compensation from the iliopsoas, oblique, and contralateral rectus abdominis muscles. However, the majority of women following free TRAM and DIEP flap reconstruction were satisfied with the postoperative abdominal strength. Statistical comparisons related to quantified abdominal strength have been previously reported..$^{9,10}$

Analysis of abdominal contour demonstrates that as the amount of rectus abdominis muscle and anterior rectus sheath excised is minimized, the incidence of abdominal bulge decreases. This has been previously demonstrated. ${ }^{33,34}$ There does not appear to be a difference when comparing the MS-3 group to the MS-2 group. The supportive aspect of the anterior abdominal wall appears to be related primarily to the integrity of the anterior rectus sheath. During flap elevation, the anterior sheath is elevated off the surface of the muscle, which may devascularize the fascia. It is postulated that preservation of the rectus abdominis muscle and its segmental blood supply and innervation will facilitate the revascularization of the anterior rectus sheath and maintain it supportive role. Attenuation of the anterior rectus sheath may be secondary to excessive harvest of the muscle, leading to poor revascularization of the fascia or to denervation of the muscle, which leads to atrophy and fibrosis that would also inhibit fascial revascularization.

On the basis of our results, the outcome following free TRAM and DIEP flap breast reconstruction is optimized by preoperative assessment of tissue requirements, intraoperative assessment of perforators, and proper patient selection. The three-dimensional imaging system is an excellent means of assessing tissue requirements. Factors that are associated with the occurrence of fat necrosis in the free TRAM or DIEP flap include patient body weight and flap volume requirements. Factors that are associated with the inability to perform sit-ups include patient body weight and age. The ability to perform sit-ups is not related to maximal preservation of the rectus abdominis muscle or intercostatinnervation. There is no difference in the ability to perform sit-ups between the free TRAM flap and the DIEP flap. Abdominal contour is improved following flap elevation using the MS-2 or MS-3 techniques when compared with/the MS-1 and MS-0 techniques.

Maurice Y. Nahabedian, M.D.
Johns Hopkins University
601 North Caroline Street, 8152C
Baltimore, Md. 21287
mahabed@jhmi.edu
REFERENCES

1. Feller, A. M. Free TRAM: Results and abdominal wall function. Clin. Plast. Surg. 21: 223, 1994.

2. Arnez, Z. M., Khan, U., Pogorelec, D., and Planinsek, F. Rational selection of flaps from the abdomen in breast reconstruction to reduce donor site morbidity. $\mathrm{Br}$. J. Plast. Surg. 52: 351, 1999.

3. Serletti, J. M., and Moran, S. L. Free versus the pedicled TRAM flap: A cost comparison and outcome analysis. Plast. Reconstr. Surg. 100: 1418, 1997.

4. Serletti, J. M., and Moran, S. L. Microvascular reconstruction of the breast. Semin. Surg. Oncol. 19: 264, 2000.

5. Kroll, S. S., Gherardini, G., Martin, J. E., et al. Fat necrosis in free and pedicled TRAM flaps. Plast. Reconstr. Surg. 102: 1502, 1998.

6. Kroll, S. S., Schusterman, M. A., Reece, G. P., Miller, M. J., Robb, G., and Evans, G. R. Abdominal wall strength, bulging, and hernia after TRAM flap breast reconstruction. Plast. Reconstr. Surg. 96: 616, 1995.

7. Kroll, S. S., and Marchi, M. Comparison of strategies for preventing abdominal wall weakness after TRAM flap breast reconstruction. Plast. Reconstr. Surg. 89: 1045, 1992.

8. Edsander-Nord, A., Jurell, G., and Wickman, M. Donorsite morbidity after pedicled or free TRAM flap surgery: A prospective and objective study. Plast. Reconstr. Surg. 102: 1508, 1998. 
9. Futter, C. M., Webster, M. H., Hagen, S., and Mitchell, $\mathrm{S}$. L. A retrospective comparison of abdominal muscle strength following breast reconstruction with a free TRAM or DIEP flap. Br. J. Plast. Surg. 53: 578, 2000.

10. Blondeel, P. N., Vanderstraeten, G. G., Monstrey, S. J., et al. The donor site morbidity of free DIEP flaps and free TRAM flaps for breast reconstruction. Br. J. Plast. Surg. 50: 322, 1997.

11. Hamdi, M., Weiler-Mithoff, E. M., and Webster, M. H. Deep inferior epigastric perforator flap in breast reconstruction: Experience with the first 50 flaps. Plast. Reconstr. Surg. 103: 86, 1999.

12. Blondeel, P. N., Arnstein, M., Verstraete, K., et al. IVenous congestion and blood flow in free transverse rectus abdominis myocutaneous and deep inferior epigastric perforator flaps. Plast. Reconstr. Surg. 106: 1295, 2000.

13. Blondeel, P. N. One hundred free DIEP flap breast reconstructions: A personal experience. Br. J. Plast. Surg. 52: 104, 1999.

14. Kroll, S. S. Fat necrosis in free transverse rectus abdominis myocutaneous and deep inferior epigastric perforator flaps. Plast. Reconstr. Surg. 106: 576, 2000.

15. Allen, R. J., and Treece, P. Deep inferior epigastric perforator flap for breastreconstruction. Ann. Plast. Surg. 32: 32, 1994.

16. Dupin, C. L., Allen, R. J., Glass, C.A., and Bunch, R. The internal mammary artery and vein as a recipient site for free flap breast reconstruction: A report of 110 consecutive cases. Plast. Reconstr. Surg. 98: 685, 1996.

17. Blondeel, P. N., and Boeckx, W. D. Refinements in free flap breast reconstruction: The free bilateral deep inferior epigastric perforator flap anastomosed to the internal mammary artery. Br. J. Plast. Surg. 47: 495, 1994.

18. Grotting, J. C., Urist, M. M., Maddox, W. A., and Vasconez, L. O. Conventional TRAM versus free microsurgical TRAM flap for immediate breast reconstruction. Plast. Reconstr. Surg. 83: 828, 1989.

19. Heitmann, C., Felmerer, G., Durmus, C., Matejic, B., and Ingianni, G. Anatomic features of perforator blood vessels in the deep inferior epigastric perforator flap. Br. J. Plast. Surg. 53: 205, 2000.

20. Blondeel, P. N., Beyens, G., Verhaeghe, R., et al. Doppler flowmetry in the planning of perforator flaps. Br.J. Plast. Surg. 51: 202, 1998.

21. Chang, B. W., Luethke, R., Berg, W. A., Hamper, U. M., and Manson, P. N. Two-dimensional color Doppler imaging for precision preoperative mapping and size determination of TRAM flap perforators. Plast. Reconstr. Surg. 93: 197, 1994.
22. Rand, R. P., Cramer, M. M., and Strandness, D. E., Jr. Color-flow duplex scanning in the preoperative assessment of TRAM flap perforators: A report of 32 consecutive patients. Plast. Reconstr. Surg. 93: 453, 1994.

23. Berg, W. A., Chang, B. W., DeJong, M. R., and Hamper, U. M. Color Doppler flow mapping of abdominal wall perforating arteries for transverse rectus abdominis myocutaneous flap in breast reconstruction: Method and preliminary results. Radiology 192: 447, 1994.

24. Chang, D. W., Reece, G. P., Wang, B., et al. Effect of smoking on complications in patients undergoing free TRAM flap breast reconstruction. Plast. Reconstr. Surg. 105; 2374, 2000.

25. Chang, D. W., Wang, B., Robb, G. L., et al. Effect of obesity on flap and donor site complications in free transverse rectus abdominis myocutaneous flap breast reconstruction. Plast. Reconstr. Surg. 105: 1640, 2000.

26. Kroll, S. S. Bilateral breast reconstruction in very thin - patients with extended free TRAM flaps. Br. J. Plast. Surg. 51: 535, 1998.

27. Kroll, S. S., Schusterman, M. A., Reece, G. P., et al. Choice of flap and incidence of free flap success. Plast. Reconstr. Surg. 98: 459, 1996.

28. Evans, G. R., and Kroll, S. S. Choice of technique for reconstruction.Clin. Plast. Surg. 25: 311, 1998.

29. Galdino, G. M., Nahabedian, M. Y., Chang, B. W., et al. Three dimensional digital photography of the breast: Clinical applications. In Proceedings from the Annual Meeting of the American Society of Plastic Surgeons, Los Angeles, Calif., October 14-18, 2000.

30. Duchateau, J., Declety, A., and Lejour, M. Innervation of the rectus abdominis muscle: Implications for rectus flaps. Plast. Reconstr. Surg. 82: 223, 1988.

31. Hammond, D. C., Larson, D. L., Severinac, R. N., and Marcias, M. Rectus abdominis muscle innervation: Implications for TRAM flap elevation. Plast. Reconstr. Surg. 96: 105, 1995.

32. SAS Institute, Inc. SAS/STAT User's Guides: Online Documentation. Cary, N.C.: SAS Institute, Inc., 1999.

33. Nahabedian, M. Y., and Manson, P. M. Contour abnormalities of the abdomen following TRAM flap breast reconstruction: A multifactorial analysis. Plast. Reconstr. Surg. 109: 81, 2002.

34. Nahabedian, M. Y., Dooley, W., Singh, N., and Manson, P. M. Contour abnormalities of the abdomen following breast reconstruction with abdominal flaps: The role of muscle preservation. Plast. Reconstr. Surg. 109: 91, 2002. 